\title{
Solvent-free synthesis and assessment of substituent effects from IR \& NMR spectra of some substituted benzylidene-5-ethyl-1,3,4-thiadiazole-2-amines
}

\author{
G. Thirunarayanan \\ Department of Chemistry, Annamalai University, Annamalainagar - 608002, India \\ E-mail address: drgtnarayanan@gmail.com
}

\begin{abstract}
A series containing ten titled compounds were synthesized by $\mathrm{SiO}_{2}: \mathrm{H}_{3} \mathrm{PO}_{4}$ catalyzed solventfree condensation of substituted benzaldehydes and 2-amino-5-ethyl-1,3,4-thiadiazole under microwave irradiation. The yields of prepared amines are more than $90 \%$. The synthesized amines were characterized by their physical constants and spectroscopic data reported in literature earlier for known compounds. The assigned spectral group frequencies such as infrared $v \mathrm{C}=\mathrm{N}, \mathrm{C}-\mathrm{S}-\mathrm{C}, \mathrm{N}-\mathrm{N}\left(\mathrm{cm}^{-}\right.$ $\left.{ }^{1}\right)$ and NMR chemical shifts $(\delta, \mathrm{ppm})$ of $\mathrm{CH}, \mathrm{C}=\mathrm{N}$ have been correlated with Hammett substituent constants, $\mathrm{F}$ and $\mathrm{R}$ parameters using single and multi-linear regression analysis. From the results of statistical analyses, the effects of substituent on the above spectral data have been discussed.
\end{abstract}

Keywords: Benzylidene-5-ethyl-1,3,4-thiadiazole-2-amines; Solvent-free synthesis; $\mathrm{SiO}_{2}: \mathrm{H}_{3} \mathrm{PO}_{4}$; IR spectra; NMR spectra; Hammett correlation

\section{INTRODUCTION}

The five membered rings containing one sulphor atom, two nitrogen atoms vicinal pattern with two double bonds are known as thiadiazoles. These thiadiazoles were medicinally important due to the presence of $\mathrm{C}=\mathrm{N}, \mathrm{C}-\mathrm{S}-\mathrm{C}$ and $\mathrm{N}-\mathrm{N}$ moieties in the five membered ring. Therefore chemists and researchers paid more attentions for synthesis of heterocyclic compounds by synthetic routes or naturally occurring methods [1]. Some of the important biological activities of these thiadiazoles are antitumor agent [2], potent inhibitors of 5-lipoxygenase and cyclooxygenase [3], antimicrobials [4], anti-tuberculosis [5,6], antiinflammatory [7], antidepressant and anxiolytics [8], anticancer [9,10], anthelmintic [11] and antimicrobial [1]. Numerous solvent-free methods and solvent assisted methods were reported for the synthesis of heterocyclics [1,12-14]. Currently, organic chemists and researchers paid much more interest for solvent-free synthetic methods due to easy handling procedure, technique, non-hazardousness, pollution free environment, shorter reaction time and better yields [16-19].

Spectroscopic data are most useful for predicting the ground state equilibration of alkenes [20], alkynes [21], chalcones [22], Troger bases [23], bis-imines [24], sulfonamides [25], thienyl-3-carboxamides [26], acyl halides and its esters [27]. The spectral data like UV 
absorption $(\lambda \max , \mathrm{nm})$, IR stretches $v\left(\mathrm{~cm}^{-1}\right)$ and NMR proton and carbon chemical shifts $(\delta$, $\mathrm{ppm}$ ) were used for the study of effect of substituent on the spectral group frequencies of organic substrates $[28,29]$.

Recently, Thirunarayanan and Sekar studied the effect of substituents on the pyrazoline derivatives using IR and NMR data [30]. Thirunarayanan have studied the solvent-free synthesis and spectral correlation of some six membered heterocyclic compounds [19]. Suresh et al., have studied the solvent-free synthesis and spectral correlations of some aryl $E$-imines [18].

Thirunarayanan et al. have studied the solvent-free synthesis and spectral linearity of some trifluoromethyl imines [31]. Sakthinathan et al., have studied the Spectral correlations and antimicrobial activities of some (E)-N-Benzylidenepyridin-2-amines [32] and aryl (E)-Nbenzylidene-3-nitrobenzenamines [33]. Within the above view, there is no report available for the solvent-free synthesis and the study of effect of substituents on the substituted benzylidene-5-ethyl-1,3,4-thiadiazole-2-amines in the past and present. Therefore, first time, the author wishes to report the solvent-free synthesis of some substituted benzylidene-5ethyl-1,3,4-thiadiazole-2-amines and recorded the IR and NMR spectra for studying the effect of substituents on the spectral frequencies.

\section{EXPERIMENTAL}

\section{1. General}

Chemical used in this present study were procured from Sigma-Aldrich and E-Merck chemical companies. The melting point of all compounds were determined in in open glass capillaries on V-SCIENTIFIC MP-DS melting point apparatus and are uncorrected. The infrared spectra of all compounds were recorded in $\mathrm{KBr}$ discs on Thermo scientific Nicolet iS5, US made Fourier transform spectrophotometer.

The NMR spectra of selective compounds were recorded in BRUKER AV 400 spectrometer operating at $400 \mathrm{MHz}$ for ${ }^{1} \mathrm{H}$ NMR spectra and $100 \mathrm{MHz}$ for ${ }^{13} \mathrm{C}$ NMR spectra in $\mathrm{CDCl}_{3}$ solvent using TMS as internal standard. Mass spectra of all synthesised thiadiazoles-2-amines were recorded on SHIMADZU mass spectrometer using chemical ionization technique.

\section{2. General procedure for synthesis of substituted}

\section{benzylidene-5-ethyl-1,3,4-thiadiazole-2-amines}

An equi-molar quantities of 2-amino-5-ethyl-1,3,4-thiadiazole (1 mmol), substituted benzaldehydes $(1 \mathrm{mmol})$ and $\mathrm{SiO}_{2}: \mathrm{H}_{3} \mathrm{PO}_{4}(0.4 \mathrm{~g})$ were taken in a $50 \mathrm{~mL}$ borosil beaker and closed with watch glass. These contents were subjected to microwave irradiation for 2-5 minutes at $650 \mathrm{~W}$ (Scheme 1) (Samsung, Microwave Oven, 100-700 W). After completion of the reaction, dichloromethane $(20 \mathrm{~mL})$ was added, followed by simple filtration.

The solution was concentrated and purified by re-crystallization. The synthesized thiadiazoles-2-amines were characterized by their physical constants, IR, ${ }^{1} \mathrm{H}$ and ${ }^{13} \mathrm{C} \mathrm{NMR}$ and Mass spectral data. The analytical, physical constants and mass fragments $(\mathrm{m} / \mathrm{z})$ data are presented in Table 1. 


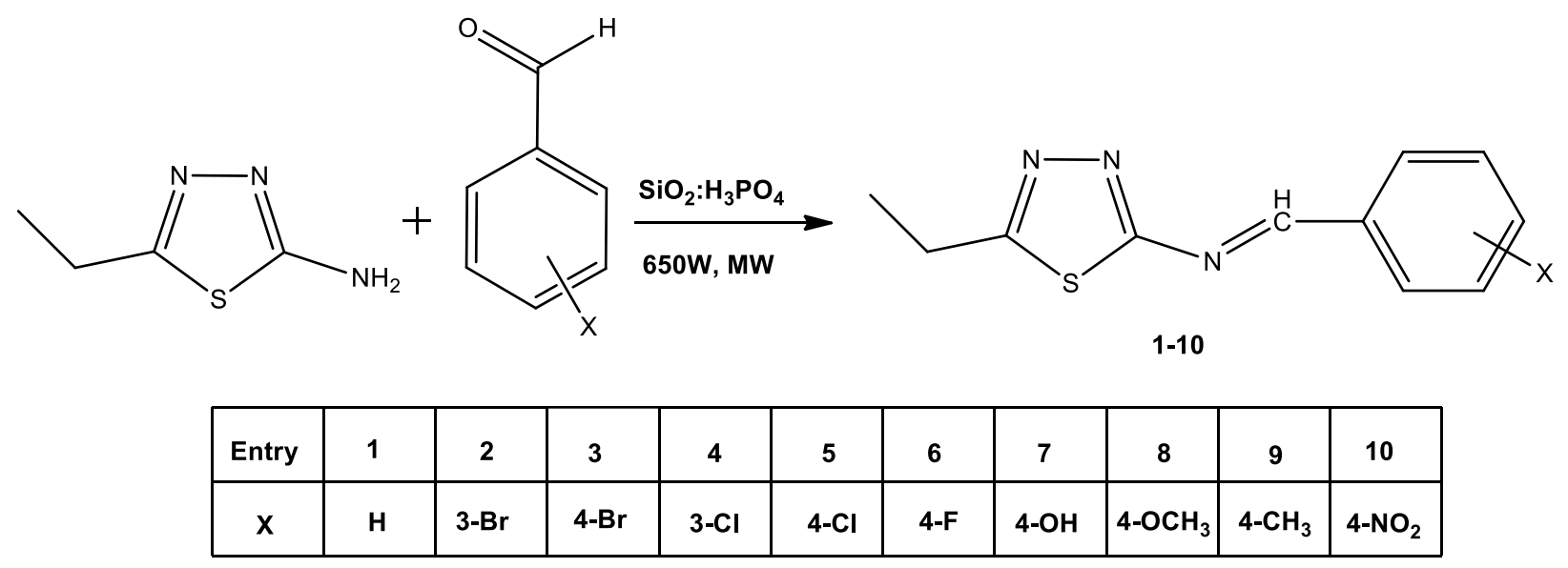

Scheme 1. Solvent-free synthesis of substituted benzylidene-5-ethyl-1,3,4-thiadiazole-2-amines.

Table 1. Physical constants, analytical and mass fragments $(\mathrm{m} / \mathrm{z})$ of substituted benzylidene-5-ethyl-1,3,4-thiadiazole-2-amines.

\begin{tabular}{|c|c|c|c|c|c|c|}
\hline Entry & $\mathbf{X}$ & M. F. & M.W. & Yield (\%) & m.p. ${ }^{\circ} \mathrm{C}$ & $\operatorname{Mass}(\mathbf{m} / \mathbf{z})$ \\
\hline 1 & $\mathrm{H}$ & $\mathrm{C}_{11} \mathrm{H}_{12} \mathrm{~N}_{3} \mathrm{~S}$ & 217 & 93 & $\begin{array}{c}234-235 \\
(231-234)[1]\end{array}$ & $217\left[\mathrm{M}^{+}\right]$ \\
\hline 2 & $3-\mathrm{Br}$ & $\mathrm{C}_{11} \mathrm{H}_{11} \mathrm{BrN}_{3} \mathrm{~S}$ & 296 & 90 & $117-118$ & $296\left[\mathrm{M}^{+}\right], 298\left[\mathrm{M}^{2+}\right]$ \\
\hline 3 & $4-\mathrm{Br}$ & $\mathrm{C}_{11} \mathrm{H}_{11} \mathrm{BrN}_{3} \mathrm{~S}$ & 296 & 90 & $\begin{array}{c}145-146 \\
(143-146)[1]\end{array}$ & $296\left[\mathrm{M}^{+}\right], 298\left[\mathrm{M}^{2+}\right]$ \\
\hline 4 & $3-\mathrm{Cl}$ & $\mathrm{C}_{11} \mathrm{H}_{11} \mathrm{ClN}_{3} \mathrm{~S}$ & 252 & 90 & $134-135$ & $252\left[\mathrm{M}^{+}\right], 254\left[\mathrm{M}^{2+}\right]$ \\
\hline 5 & $4-\mathrm{Cl}$ & $\mathrm{C}_{11} \mathrm{H}_{11} \mathrm{ClN}_{3} \mathrm{~S}$ & 252 & 91 & $\begin{array}{c}126-128 \\
(124-127)[1]\end{array}$ & $252\left[\mathrm{M}^{+}\right], 254\left[\mathrm{M}^{2+}\right]$ \\
\hline 6 & $4-\mathrm{F}$ & $\mathrm{C}_{11} \mathrm{H}_{11} \mathrm{FN}_{3} \mathrm{~S}$ & 235 & 90 & $\begin{array}{c}172-173 \\
(167-171)[1]\end{array}$ & $235\left[\mathrm{M}^{+}\right], 237\left[\mathrm{M}^{2+}\right]$ \\
\hline 7 & $4-\mathrm{OH}$ & $\mathrm{C}_{11} \mathrm{H}_{12} \mathrm{~N}_{3} \mathrm{OS}$ & 233 & 92 & $\begin{array}{c}187-188 \\
(187-192)[1]\end{array}$ & $233\left[\mathrm{M}^{+}\right]$ \\
\hline 8 & $4-\mathrm{OCH}_{3}$ & $\mathrm{C}_{12} \mathrm{H}_{14} \mathrm{~N}_{3} \mathrm{OS}$ & 247 & 94 & $\begin{array}{c}144-145 \\
(142-144)[1]\end{array}$ & $247\left[\mathrm{M}^{+}\right]$ \\
\hline 9 & $4-\mathrm{CH}_{3}$ & $\mathrm{C}_{12} \mathrm{H}_{14} \mathrm{~N}_{3} \mathrm{~S}$ & 231 & 93 & $\begin{array}{c}205-206 \\
(204-207)[1]\end{array}$ & $231\left[\mathrm{M}^{+}\right]$ \\
\hline 10 & $4-\mathrm{NO}_{2}$ & $\mathrm{C}_{11} \mathrm{H}_{11} \mathrm{~N}_{4} \mathrm{O}_{2} \mathrm{~S}$ & 262 & 90 & $\begin{array}{c}139-140 \\
(134-138)[1]\end{array}$ & $262\left[\mathrm{M}^{+}\right]$ \\
\hline
\end{tabular}




\section{RESULTS AND DISCUSSION}

In the present investigation, the author have synthesized and recorded IR and NM spectra for studying the effect of substituents on the spectral frequencies of substituted benzylidene-5-ethyl-1,3,4-thiadiazole-2-amines by infrared and NMR spectra.

\section{1. IR spectral study}

The infrared spectra of all substituted benzylidene-5-ethyl-1,3,4-thiadiazole-2-amines were recorded in $\mathrm{KBr}$ discs. The characteristic stretches of the substituted benzylidene-5ethyl-1,3,4-thiadiazole-2-amines such as $v \mathrm{C}=\mathrm{N}, \mathrm{C}-\mathrm{S}-\mathrm{C}, \mathrm{N}-\mathrm{N}\left(\mathrm{cm}^{-1}\right)$ were assigned and tabulated in Table 2. These stretches were correlated with Hammett substituent constants, F and $\mathrm{R}$ parameters [34] using linear regression analysis. In this correlation, the Hammett equation is employed in the form as,

$$
v=\rho \sigma+v_{\mathrm{o}}
$$

where $v$ is the carbonyl frequencies of substituted system and $v_{0}$ is the corresponding quantity of unsubstitued system, $\sigma$ is a Hammett substituent constant, which in principle is characteristics of the substituent and $\rho$ is a reaction constant which is depend upon the nature of the reaction. The results of statistical analyses [12,18,19,22-24,27-29,32] was presented in Table 3.

Table 2. IR and NMR spectral data of substituted benzylidene-5-ethyl-1,3,4-thiadiazole-2-amines.

\begin{tabular}{|c|c|c|c|c|c|c|}
\hline \multirow{2}{*}{ Entry } & \multirow{2}{*}{$\mathbf{X}$} & \multicolumn{3}{|c|}{ IR $\mathbf{~ ( \mathbf { c m } ^ { - \mathbf { 1 } } )}$} & ${ }^{\mathbf{1}}$ H NMR $\boldsymbol{\delta}(\mathbf{p p m})$ & ${ }^{13}$ C NMR $\boldsymbol{\delta}(\mathbf{p p m})$ \\
\cline { 3 - 7 } & & $\mathrm{C}=\mathrm{N}$ & $\mathrm{C}-\mathrm{S}-\mathrm{C}$ & $\mathrm{N}-\mathrm{N}$ & $\mathrm{CH}=\mathrm{N}$ & $\mathrm{CH}=\mathrm{N}$ \\
\hline $\mathbf{1}$ & $\mathrm{H}$ & 1578 & 645 & 1030 & 8.215 & 161.35 \\
\hline $\mathbf{2}$ & $3-\mathrm{Br}$ & 1576 & 652 & 1036 & 8.232 & 161.58 \\
\hline $\mathbf{3}$ & $4-\mathrm{Br}$ & 1576 & 643 & 1020 & 8.129 & 161.33 \\
\hline $\mathbf{4}$ & $3-\mathrm{Cl}$ & 1571 & 641 & 1025 & 8.124 & 161.36 \\
\hline $\mathbf{5}$ & $4-\mathrm{Cl}$ & 1579 & 649 & 1040 & 8.089 & 161.29 \\
\hline $\mathbf{6}$ & $4-\mathrm{F}$ & 1581 & 646 & 1032 & 8.421 & 161.49 \\
\hline $\mathbf{7}$ & $4-\mathrm{OH}$ & 1573 & 650 & 1039 & 8.125 & 161.04 \\
\hline $\mathbf{8}$ & $4-\mathrm{OCH}_{3}$ & 1572 & 640 & 1018 & 8.002 & 161.02 \\
\hline $\mathbf{9}$ & $4-\mathrm{CH}_{3}$ & 1574 & 648 & 1031 & 8.236 & 161.89 \\
\hline $\mathbf{1 0}$ & $4-\mathrm{NO}_{2}$ & 1583 & 650 & 1037 & 8.625 & \\
\hline
\end{tabular}


From the Table 3, The correlation of $v \mathrm{C}=\mathrm{N}\left(\mathrm{cm}^{-1}\right)$ stretches with Hammett $\sigma, \sigma^{+}$ substituent constants have shown satisfactory $r$ values $\left(\sigma: r=0.954, \sigma^{+}: r=0.951\right)$. The Hammett $\sigma_{I}, \sigma_{R}, F$ and R parameters correlated satisfactorily $\left(\sigma_{I}: r=0.904, \sigma_{R}: r=903, F: r\right.$ $=0.904, \mathrm{R}=0.904$ ) excluding $\mathrm{H}, 4-\mathrm{F}$ and $4-\mathrm{CH}_{3}$ substituents. If these substituents were included in the correlation, they reduced the $\mathrm{r}$ values significantly. All correlations gave positive $\rho$ values. This implies that the normal substituent effect operates in all system.

The $v C-S-C\left(\mathrm{~cm}^{-1}\right)$ and $v N-N\left(\mathrm{~cm}^{-1}\right)$ stretches of substituted benzylidene-5-ethyl-1,3,4thiadiazole-2-amines were fail in correlation with Hammett substituent constants, $\mathrm{F}$ and $\mathrm{R}$ parameters. All correlations gave positive $\rho$ values. This implies that the normal substituent effect operates in all system. The failure in correlation is due to the inability of the effect of substituents on the group frequencies and associated with resonance conjugative structure as shown in Fig. 1.

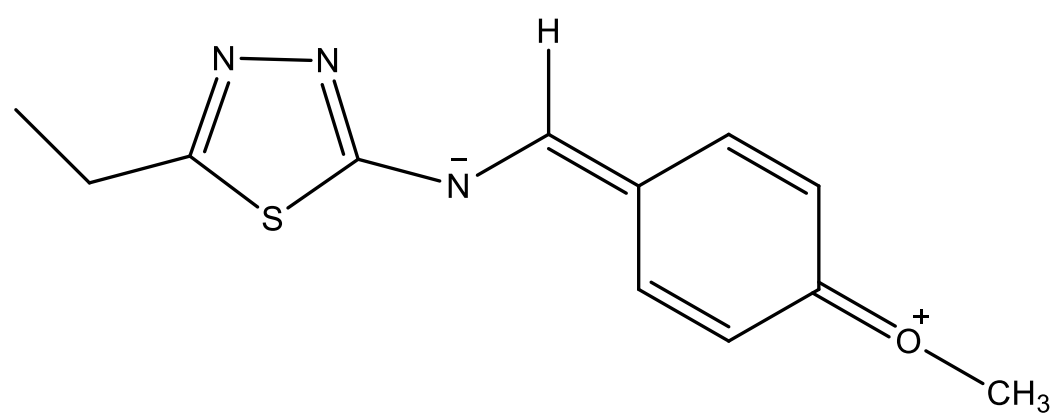

Fig. 1. Resonance-conjugative structure.

In infrared spectral correlation of substituted benzylidene-5-ethyl-1,3,4-thiadiazole-2amines, the correlation of $v \mathrm{C}-\mathrm{S}-\mathrm{C}\left(\mathrm{cm}^{-1}\right)$ and $v \mathrm{~N}-\mathrm{N}\left(\mathrm{cm}^{-1}\right)$ stretches with Hammett substituent constants, $\mathrm{F}$ and $\mathrm{R}$ parameters were fail in single parameter regression analysis. These data gave satisfactory correlations, when they worthful, while seeking in multi-regression analysis with $\sigma_{\mathrm{I}}$ and $\sigma_{\mathrm{R}}$ or $\mathrm{F}$ and $\mathrm{R}$ parameters [34]. The generated multi-regression analysis equations are given in (2-7).

Table 3. Results of statistical analysis of IR, ${ }^{1} \mathrm{H}$ NMR, ${ }^{13} \mathrm{C}-\mathrm{NMR}$ and UV spectral values of substituted benzylidene-5-ethyl-1,3,4-thiadiazole-2-amineswith Hammett $\sigma, \sigma^{+}, \sigma_{\mathrm{I}}, \sigma_{\mathrm{R}}$ constants, F and $\mathrm{R}$ parameters.

\begin{tabular}{|c|c|c|c|c|c|c|c|}
\hline Frequency & Constant & $\mathbf{r}$ & I & $\boldsymbol{\rho}$ & $\mathbf{s}$ & $\mathbf{n}$ & Correlated derivatives \\
\hline \multirow{5}{*}{ 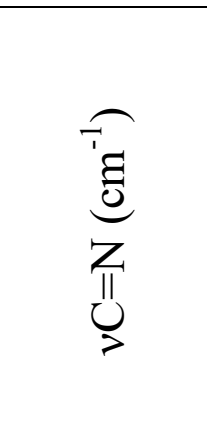 } & $\sigma$ & 0.954 & 1575.52 & 9.179 & 3.49 & 10 & $\begin{array}{l}\mathrm{H}, 3-\mathrm{Br}, 4-\mathrm{Br}, 3-\mathrm{Cl}, 4-\mathrm{Cl}, 4-\mathrm{F}, \\
4-\mathrm{OH}, 4-\mathrm{OCH}_{3}, 4-\mathrm{CH}_{3}, 4-\mathrm{NO}_{2}\end{array}$ \\
\hline & $\sigma^{+}$ & 0.951 & 1576.28 & 3.761 & 3.58 & 10 & $\begin{array}{l}\mathrm{H}, 3-\mathrm{Br}, 4-\mathrm{Br}, 3-\mathrm{Cl}, 4-\mathrm{Cl}, 4-\mathrm{F}, \\
4-\mathrm{OH}, 4-\mathrm{OCH}_{3}, 4-\mathrm{CH}_{3}, 4-\mathrm{NO}_{2}\end{array}$ \\
\hline & $\sigma_{\mathrm{I}}$ & 0.904 & 1573.66 & 7.410 & 3.78 & 8 & $\begin{array}{c}\text { 3-Br, 4-Br, 3-Cl, 4-Cl, 4-F, } \\
\text { 4-OH, 4- } \mathrm{OCH}_{3}, 4-\mathrm{NO}_{2}\end{array}$ \\
\hline & $\sigma_{\mathrm{R}}$ & 0.903 & 1577.83 & 7.810 & 3.84 & 9 & $\begin{array}{c}\mathrm{H}, 3-\mathrm{Br}, 4-\mathrm{Br}, 3-\mathrm{Cl}, 4-\mathrm{Cl} \\
\text { 4-OH, 4-- }\end{array}$ \\
\hline & $\mathrm{F}$ & 0.904 & 1573.25 & 8.106 & 3.65 & 8 & $\begin{array}{c}\text { 3-Br, 4- } \mathrm{Br}, 3-\mathrm{Cl}, 4-\mathrm{Cl}, 4-\mathrm{F}, \\
4-\mathrm{OH}, 4-\mathrm{OCH}_{3}, 4-\mathrm{NO}_{2}\end{array}$ \\
\hline
\end{tabular}




\begin{tabular}{|c|c|c|c|c|c|c|c|}
\hline & $\mathrm{R}$ & 0.904 & 1577.93 & 5.983 & 3.82 & 9 & $\begin{array}{c}\mathrm{H}, 3-\mathrm{Br}, 4-\mathrm{Br}, 3-\mathrm{Cl}, 4-\mathrm{Cl}, \\
\text { 4-OH, 4-OCH }, 4-\mathrm{CH}_{3}, 4-\mathrm{NO}_{2}\end{array}$ \\
\hline \multirow{6}{*}{ 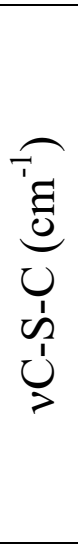 } & $\sigma$ & 0.823 & 646.05 & 2.752 & 4.21 & 10 & $\begin{array}{l}\mathrm{H}, 3-\mathrm{Br}, 4-\mathrm{Br}, 3-\mathrm{Cl}, 4-\mathrm{Cl}, 4-\mathrm{F}, \\
4-\mathrm{OH}, 4-\mathrm{OCH}_{3}, 4-\mathrm{CH}_{3}, 4-\mathrm{NO}_{2}\end{array}$ \\
\hline & $\sigma^{+}$ & 0.815 & 646.39 & 1.146 & 4.28 & 10 & $\begin{array}{l}\mathrm{H}, 3-\mathrm{Br}, 4-\mathrm{Br}, 3-\mathrm{Cl}, 4-\mathrm{Cl}, 4-\mathrm{F}, \\
4-\mathrm{OH}, 4-\mathrm{OCH}_{3}, 4-\mathrm{CH}_{3}, 4-\mathrm{NO}_{2}\end{array}$ \\
\hline & $\sigma_{\mathrm{I}}$ & 0.812 & 645.60 & 2.252 & 4.30 & 10 & $\begin{array}{l}\mathrm{H}, 3-\mathrm{Br}, 4-\mathrm{Br}, 3-\mathrm{Cl}, 4-\mathrm{Cl}, 4-\mathrm{F} \\
4-\mathrm{OH}, 4-\mathrm{OCH}_{3}, 4-\mathrm{CH}_{3}, 4-\mathrm{NO}_{2}\end{array}$ \\
\hline & $\sigma_{\mathrm{R}}$ & 0.847 & 647.39 & 5.083 & 4.19 & 10 & $\begin{array}{l}\mathrm{H}, 3-\mathrm{Br}, 4-\mathrm{Br}, 3-\mathrm{Cl}, 4-\mathrm{Cl}, 4-\mathrm{F}, \\
4-\mathrm{OH}, 4-\mathrm{OCH}_{3}, 4-\mathrm{CH}_{3}, 4-\mathrm{NO}_{2}\end{array}$ \\
\hline & $\mathrm{F}$ & 0.817 & 645.50 & 2.373 & 4.29 & 10 & $\begin{array}{l}\mathrm{H}, 3-\mathrm{Br}, 4-\mathrm{Br}, 3-\mathrm{Cl}, 4-\mathrm{Cl}, 4-\mathrm{F}, \\
\text { 4-OH, 4-OCH } 3,4-\mathrm{CH}_{3}, 4-\mathrm{NO}_{2}\end{array}$ \\
\hline & $\mathrm{R}$ & 0.815 & 647.05 & 2.406 & 4.28 & 10 & $\begin{array}{l}\mathrm{H}, 3-\mathrm{Br}, 4-\mathrm{Br}, 3-\mathrm{Cl}, 4-\mathrm{Cl}, 4-\mathrm{F}, \\
4-\mathrm{OH}, 4-\mathrm{OCH}_{3}, 4-\mathrm{CH}_{3}, 4-\mathrm{NO}_{2}\end{array}$ \\
\hline \multirow{6}{*}{ 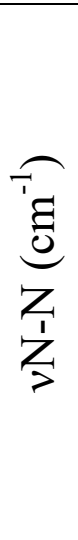 } & $\sigma$ & 0.820 & 1030.23 & 4.545 & 7.99 & 10 & $\begin{array}{c}\mathrm{H}, 4-\mathrm{Br}, 4-\mathrm{Cl}, 4-\mathrm{F}, 2-\mathrm{OH}, 2-\mathrm{OCH}_{3}, \\
\quad 4-\mathrm{OCH}_{3}, 4-\mathrm{CH}_{3}, 3-\mathrm{NO}_{2}, 4-\mathrm{NO}_{2}\end{array}$ \\
\hline & $\sigma^{+}$ & 0.809 & 1030.79 & 1.349 & 8.12 & 10 & $\begin{array}{l}\mathrm{H}, 3-\mathrm{Br}, 4-\mathrm{Br}, 3-\mathrm{Cl}, 4-\mathrm{Cl}, 4-\mathrm{F} \\
\text { 4-OH, 4-OCH } \\
\end{array}$ \\
\hline & $\sigma_{\mathrm{I}}$ & 0.814 & 1029.00 & 5.096 & 8.07 & 10 & $\begin{array}{l}\mathrm{H}, 3-\mathrm{Br}, 4-\mathrm{Br}, 3-\mathrm{Cl}, 4-\mathrm{Cl}, 4-\mathrm{F}, \\
4-\mathrm{OH}, 4-\mathrm{OCH}_{3}, 4-\mathrm{CH}_{3}, 4-\mathrm{NO}_{2}\end{array}$ \\
\hline & $\sigma_{\mathrm{R}}$ & 0.816 & 1032.05 & 6.450 & 8.05 & 10 & $\begin{array}{l}\mathrm{H}, 3-\mathrm{Br}, 4-\mathrm{Br}, 3-\mathrm{Cl}, 4-\mathrm{Cl}, 4-\mathrm{F}, \\
\text { 4-OH, 4-OCH } 3,4-\mathrm{CH}_{3}, 4-\mathrm{NO}_{2}\end{array}$ \\
\hline & $\mathrm{F}$ & 0.817 & 1028.70 & 5.563 & 8.04 & 10 & $\begin{array}{l}\mathrm{H}, 3-\mathrm{Br}, 4-\mathrm{Br}, 3-\mathrm{Cl}, 4-\mathrm{Cl}, 4-\mathrm{F}, \\
\text { 4-OH, 4- } \mathrm{OCH}_{3}, 4-\mathrm{CH}_{3}, 4-\mathrm{NO}_{2}\end{array}$ \\
\hline & $\mathrm{R}$ & 0.811 & 1031.68 & 3.242 & 8.11 & 10 & $\begin{array}{l}\mathrm{H}, 3-\mathrm{Br}, 4-\mathrm{Br}, 3-\mathrm{Cl}, 4-\mathrm{Cl}, 4-\mathrm{F} \\
4-\mathrm{OH}, 4-\mathrm{OCH}_{3}, 4-\mathrm{CH}_{3}, 4-\mathrm{NO}_{2}\end{array}$ \\
\hline \multirow{6}{*}{ 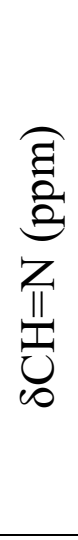 } & $\sigma$ & 0.960 & 8.180 & 0.313 & 0.15 & 10 & $\begin{array}{l}\mathrm{H}, 3-\mathrm{Br}, 4-\mathrm{Br}, 3-\mathrm{Cl}, 4-\mathrm{Cl}, 4-\mathrm{F} \\
4-\mathrm{OH}, 4-\mathrm{OCH}_{3}, 4-\mathrm{CH}_{3}, 4-\mathrm{NO}_{2}\end{array}$ \\
\hline & $\sigma^{+}$ & 0.953 & 8.219 & 0.176 & 0.16 & 10 & $\begin{array}{l}\mathrm{H}, 3-\mathrm{Br}, 4-\mathrm{Br}, 3-\mathrm{Cl}, 4-\mathrm{Cl}, 4-\mathrm{F}, \\
\text { 4-OH, 4-OCH } 3,4-\mathrm{CH}_{3}, 4-\mathrm{NO}_{2}\end{array}$ \\
\hline & $\sigma_{\mathrm{I}}$ & 0.903 & 8.108 & 0.312 & 0.17 & 8 & $\begin{array}{c}\text { 3-Br, 4-Br, 3-Cl, 4-Cl, 4-F, } \\
\text { 4-OH, 4- } \mathrm{OCH}_{3}, 4-\mathrm{NO}_{2}\end{array}$ \\
\hline & $\sigma_{\mathrm{R}}$ & 0.904 & 8.354 & 0.410 & 0.17 & 9 & $\begin{array}{c}\mathrm{H}, 3-\mathrm{Br}, 4-\mathrm{Br}, 3-\mathrm{Cl}, 4-\mathrm{Cl}, \\
\text { 4-OH, 4-OCH } 3,4-\mathrm{CH}_{3}, 4-\mathrm{NO}_{2}\end{array}$ \\
\hline & $\mathrm{F}$ & 0.904 & 8.080 & 0.362 & 0.16 & 8 & $\begin{array}{l}\text { 3- } \mathrm{Br}, 4-\mathrm{Br}, 3-\mathrm{Cl}, 4-\mathrm{Cl}, 4-\mathrm{F}, \\
\text { 4-OH, 4- } \mathrm{OCH}_{3}, 4-\mathrm{NO}_{2}\end{array}$ \\
\hline & $\mathrm{R}$ & 0.904 & 8.296 & 0.779 & 0.17 & 8 & $\begin{array}{l}\mathrm{H}, 3-\mathrm{Br}, 4-\mathrm{Br}, 3-\mathrm{Cl}, 4-\mathrm{Cl} \\
4-\mathrm{OCH}_{3}, 4-\mathrm{CH}_{3}, 4-\mathrm{NO}_{2}\end{array}$ \\
\hline \multirow{6}{*}{ 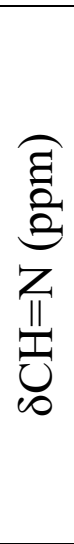 } & $\sigma$ & 0.937 & 161.47 & 0.282 & 0.74 & 10 & $\begin{array}{l}\mathrm{H}, 3-\mathrm{Br}, 4-\mathrm{Br}, 3-\mathrm{Cl}, 4-\mathrm{Cl}, 4-\mathrm{F}, \\
4-\mathrm{OH}, 4-\mathrm{OCH}_{3}, 4-\mathrm{CH}_{3}, 4-\mathrm{NO}_{2}\end{array}$ \\
\hline & $\sigma^{+}$ & 0.921 & 161.44 & 0.113 & 0.80 & 10 & $\begin{array}{l}\mathrm{H}, 3-\mathrm{Br}, 4-\mathrm{Br}, 3-\mathrm{Cl}, 4-\mathrm{Cl}, 4-\mathrm{F}, \\
\text { 4-OH, 4--OCH } 3,4-\mathrm{CH}_{3}, 4-\mathrm{NO}_{2}\end{array}$ \\
\hline & $\sigma_{\mathrm{I}}$ & 0.937 & 161.29 & 0.433 & 0.74 & 8 & $\begin{array}{c}\text { 3-Br, 4-Br, 3-Cl, 4-Cl, 4-F, } \\
\text { 4-OH, 4- } \mathrm{OCH}_{3}, 4-\mathrm{NO}_{2}\end{array}$ \\
\hline & $\sigma_{\mathrm{R}}$ & 0.904 & 161.277 & 0.451 & 0.75 & 9 & $\begin{array}{c}\mathrm{H}, 3-\mathrm{Br}, 4-\mathrm{Br}, 3-\mathrm{Cl}, 4-\mathrm{Cl} \\
\text { 4-OH, 4--OCH }, 4-\mathrm{CH}_{3}, 4-\mathrm{NO}_{2}\end{array}$ \\
\hline & $\mathrm{F}$ & 0.910 & 161.28 & 0.965 & 0.76 & 8 & $\begin{array}{c}\text { 3-Br, 4-Br, 3-Cl, 4-Cl, 4-F, } \\
\text { 4-OH, 4- } \mathrm{OCH}_{3}, 4-\mathrm{NO}_{2}\end{array}$ \\
\hline & $\mathrm{R}$ & 0.900 & 161.473 & 0.952 & 0.77 & 9 & $\begin{array}{c}\mathrm{H}, 3-\mathrm{Br}, 4-\mathrm{Br}, 3-\mathrm{Cl}, 4-\mathrm{Cl}, \\
4-\mathrm{OH}, 4-\mathrm{OCH}_{3}, 4-\mathrm{CH}_{3}, 4-\mathrm{NO}_{2}\end{array}$ \\
\hline
\end{tabular}

$\mathrm{r}=$ correlation coefficient $\mathrm{I}=$ intercept; $\rho=$ slope $\mathrm{s}=$ standard deviation; $\mathrm{n}=$ number of correlated derivatives 


$$
\begin{aligned}
& v \mathrm{C}=\mathrm{N}\left(\mathrm{cm}^{-1}\right)=1572.20( \pm 2.508)+7.415( \pm 3.522) \sigma_{\mathrm{I}}+7.282( \pm 1.605) \sigma_{\mathrm{R}} \\
& (\mathrm{r}=0.958, \mathrm{n}=10, \mathrm{P}>95 \%) \\
& v \mathrm{C}=\mathrm{N}\left(\mathrm{cm}^{-1}\right)=1574.76( \pm 2.181)+9.331( \pm 4.622) \mathrm{F}+7.214( \pm 1.412) \mathrm{R} \\
& (\mathrm{r}=0.968, \mathrm{n}=10, \mathrm{P}>95 \%) \\
& v \mathrm{C}-\mathrm{S}-\mathrm{C}\left(\mathrm{cm}^{-1}\right)=646.59( \pm 3.078)+2.255( \pm 0.654) \sigma_{\mathrm{I}}+5.087( \pm 0.741) \sigma_{\mathrm{R}} \\
& (\mathrm{r}=0.927, \mathrm{n}=10 \mathrm{P}>90 \%) \\
& \text { vC-S-C }\left(\mathrm{cm}^{-1}\right)=646.09( \pm 3.024)+2.849( \pm 6.412) \mathrm{F}+2.790( \pm 0.572) \mathrm{R} \\
& (\mathrm{r}=0.922, \mathrm{n}=10, \mathrm{P}>90 \%) \\
& v \mathrm{~N}-\mathrm{N}\left(\mathrm{cm}^{-1}\right)=1030.26( \pm 5.872)+5.065( \pm 1.245) \sigma_{\mathrm{I}}+6.459( \pm 1.416) \sigma_{\mathrm{R}} \\
& (\mathrm{r}=0.922, \mathrm{n}=10, \mathrm{P}>90 \%) \\
& v \mathrm{~N}-\mathrm{N}\left(\mathrm{cm}^{-1}\right)=1029.56( \pm 5.706)+6.254( \pm 1.209) \mathrm{F}+4.085( \pm 1.079) \mathrm{R} \\
& (r=0.922, n=10, P>90 \%)
\end{aligned}
$$

\section{2. NMR Spectral study}

\section{2. 1. ${ }^{1} \mathrm{H}$ NMR spectra}

The ${ }^{1} \mathrm{H}$ NMR chemical shift $(\delta$, ppm) of $\mathrm{CH}=\mathrm{N}$ proton of all substituted benzylidene-5ethyl-1,3,4-thiadiazole-2-amines were assigned and presented in Table 2. These chemical shifts are correlated $[12,18,19,22-24,27-29,32]$ with Hammett substituent constants, F and R parameters. In nuclear magnetic resonance spectra, the proton or the ${ }^{13} \mathrm{C}$ chemical shifts $(\delta)$ depends on the electronic environment of the nuclei concerned. The assigned vinyl proton chemical shifts (ppm) have been correlated with reactivity parameters using Hammett equation in the form of

$$
\log \delta=\log \delta_{\mathrm{o}}+\rho \sigma
$$

where $\delta_{o}$ is the chemical shift of unsubstitued ketones. $\sigma$ is a Hammett substituent constant, which in principle is characteristics of the substituent and $\rho$ is a reaction constant which is depend upon the nature of the reaction.

The results of statistical analysis were presented in Table 3. From Table 3, The Hammett $\sigma, \sigma^{+}$substituent constants have shown satisfactory $r$ values $\left(\sigma: r=0.951, \sigma^{+}: r=\right.$ 0.953). The Hammett $\sigma_{I}, \sigma_{R}, F$ and $R$ parameters correlated satisfactorily $\left(\sigma_{I}: r=0.903, \sigma_{R}: r\right.$ $=904, \mathrm{~F}: \mathrm{r}=0.904, \mathrm{R}=0.904)$ excluding $\mathrm{H}, 4-\mathrm{F}, 4-\mathrm{OH}$ and $4-\mathrm{CH}_{3}$ substituents. If these substituents were included in the correlation, they reduced the $r$ values significantly. All correlations gave positive $\rho$ values. This implies that the normal substituent effect operates in all system.

\section{2. 2. ${ }^{13} \mathrm{C}$ NMR spectra}

The ${ }^{13} \mathrm{C}$ NMR chemical shift $(\delta, \mathrm{ppm})$ of $\mathrm{CH}=\mathrm{N}$ carbon of all substituted benzylidene5-ethyl-1,3,4-thiadiazole-2-amines were assigned and presented in Table 2 . These chemical shifts are correlated [12,18,19,22-24,27-29,32] with Hammett substituent constants, F and R parameters. The results of statistical analysis was presented in Table 3. From Table 3, The Hammett Hammett $\sigma, \sigma^{+}$substituent constants have shown satisfactory $r$ values $(\sigma: r=0.932$, $\left.\sigma^{+}: \mathrm{r}=0.921\right)$. The Hammett $\sigma_{\mathrm{I}}, \sigma_{\mathrm{R}}, \mathrm{F}$ and $\mathrm{R}$ parameters correlated satisfactorily $\left(\sigma_{\mathrm{I}}: \mathrm{r}=\right.$ $0.937, \sigma_{\mathrm{R}}: \mathrm{r}=904, \mathrm{~F}: \mathrm{r}=0.910, \mathrm{R}=0.900$ ) excluding $\mathrm{H}, 4-\mathrm{F}$ and $4-\mathrm{CH}_{3}$ substituents. If these 
substituents were included in the correlation, they reduced the $r$ values significantly. All correlations gave positive $\rho$ values. This implies that the normal substituent effect operates in all system. Similarly the multi-regression analysis of the NMR chemical shifts $(\delta, \mathrm{ppm})$ of $\mathrm{CH}=\mathrm{N}$ proton and carbon of all substituted benzylidene-5-ethyl-1,3,4-thiadiazole-2-amines were satisfactorily correlated with $\sigma_{\mathrm{I}}$ and $\sigma_{\mathrm{R}}$ or $\mathrm{F}$ and R parameters [34]. The generated multiregression analysis equations are given in (9-12).

$$
\begin{gathered}
\delta \mathrm{CH}=\mathrm{N}(\mathrm{ppm})=8.189( \pm 0.113)+0.312( \pm 0.240) \sigma_{\mathrm{I}}+0.411( \pm 0.203) \sigma_{\mathrm{R}} \\
(\mathrm{r}=0.959, \mathrm{n}=10, \mathrm{P}>95 \%) \\
\delta \mathrm{CH}=\mathrm{N}(\mathrm{ppm})=8.151( \pm 0.100)+0.426( \pm 0.212) \mathrm{F}+0.336( \pm 0.189) \mathrm{R} \\
(\mathrm{r}=0.987, \mathrm{n}=10, \mathrm{P}>95 \%) \\
\delta \mathrm{C}=\mathrm{N}(\mathrm{ppm})=161.35( \pm 10.185)+0.434( \pm 0.232) \sigma_{\mathrm{I}}+0.299( \pm 0.041) \sigma_{\mathrm{R}} \\
(\mathrm{r}=0.943, \mathrm{n}=10, \mathrm{P}>90 \%) \\
\delta \mathrm{C}=\mathrm{N}(\mathrm{ppm})=161.31( \pm 0.179)+0.478( \pm 0.038) \mathrm{F}+0.161( \pm 0.034) \mathrm{R} \\
(\mathrm{r}=0.943, \mathrm{n}=10, \mathrm{P}>90 \%)
\end{gathered}
$$

\section{CONCLUSIONS}

Totally ten substituted benzylidene-5-ethyl-1,3,4-thiadiazole-2-amines were synthesized by $\mathrm{SiO}_{2}: \mathrm{H}_{3} \mathrm{PO}_{4}$ catalyzed solvent-free condensation under microwave irradiation. The yields of prepared amines are more than $90 \%$. This synthetic methodology offers environmentally benign reaction, easy work-up procedure, technique, non-hazardousness, pollution free to environment, shorter reaction time and better yields. The synthesized amines were characterized by their physical constants and spectroscopic data reported in literature earlier for known compounds. The assigned spectral group frequencies such as infrared $v \mathrm{C}=\mathrm{N}, \mathrm{C}-\mathrm{S}-\mathrm{C}, \mathrm{N}-\mathrm{N}\left(\mathrm{cm}^{-1}\right)$ and NMR chemical shifts $(\delta, \mathrm{ppm})$ of $\mathrm{CH}, \mathrm{C}=\mathrm{N}$ have been correlated with Hammett substituent constants, $\mathrm{F}$ and $\mathrm{R}$ parameters using single and multilinear regression analysis. From the results of statistical analyses, infrared $v C=N\left(\mathrm{~cm}^{-1}\right)$ and NMR chemical shifts $(\delta, \mathrm{ppm})$ of $\mathrm{CH}, \mathrm{C}=\mathrm{N}$ have shown satisfactory correlations with single and multi-regression analysis.

\section{ACKNOWLEDGEMENT}

The author thank DST-NMR facility, Department of Chemistry, Annamalai University, Annamalainagar 608002 for recording NMR spectra.

\section{References}

[1] S. Kumar, S. K. Sharma, S. Jain, Der Pharm. Chem. 5(5) (2013) 54-58.

[2] M. Barboiu, M. Cimpoesu, C. Guran, C. T. Supuran, Metal based drug 3(5) (1996) 227-232.

[3] D. H. Boschelli, D. T. Connor, D. A. Bornemeier, R. D. Dyer, J. A. Kennedy, P. J. Kuipers, G. C. Okonkwo, D. J. Schrier, C. D. Wright, J. Med. Chem. 36 (1993) 1802-1810.

[4] A. A. Aly, R. El-Sayed, Chem. Pap. 60(1) (2006) 56-60. 
[5] E. E. Orucü, S. Rollas, F. Kandemirli, N. Shvets A. S. Dimoglo, J. Med. Chem. 47 (2004) 6760-6767.

[6] T. S. Zhivotova, A. M. Zhivotova, O. V. Dryuk, A. Zh Seitembetova., Russ. J. Appl. Chem. 81(2) (2008) 259-262.

[7] J. Sainy, G. P. Mishra, R. Sharma, S. C. Chaturvedi, Pharm. Chem. J. 43(1) (2009) $19-24$.

[8] F. Clerici, D. Pocar, J. Med. Chem. 44 (2001) 931-936.

[9] T. M. Gireesh, R. R. Kamble, T. Taj, Pharm. Chem. J. 45(5) (2011) 313-316.

[10] S. Dhanya, A. M. Isloor, P. Shetty, K. Satyamoorthy, A. S. B. Prasad, Med. Chem. Res. 20 (2011) 1074-1080.

[11] N. S. Begum, D. E. Vasundhara, C. R. Giriraj, G. D. Kolavi, V. S. Hegde, I. M. Khazi, J. Chem. Crystallogr. 37(3) (2007) 193-198.

[12] G. Thirunarayanan, P. Mayavel, K. Thirumurthy, S. Dineshkumar, R. Sasikala, P. Nisha, A. Nithyaranjani, European Chem. Bull. 2(9) (2013) 598-605.

[13] K. G. Sekar, G. Thirunarayanan, International Letters of Chemistry, Physics and Astronomy 8(2) (2013) 160-174.

[14] G. Thirunarayanan, K. G. Sekar, International Letters of Chemistry, Physics and Astronomy 10(1) (2013) 18-34.

[15] G. Thirunarayanan, V. Renuka, K. G. Sekar, K. Lakshmanan, K. Anbarasu, International Letters of Chemistry, Physics and Astronomy 4 (2014) 66-81.

[16] G. Thirunarayanan, P. Mayavel, K. Thirumurthy, Spectrochim. Acta. 91A (2012) 18-22.

[17] G. Thirunarayanan, R. Arulkumaran, R. Sundararajan, International Letters of Chemistry, Physics and Astronomy 4 (2014) 82-97.

[18] R. Suresh, D. Kamalakkannan, K. Ranganathan, R. Arulkumaran, R. Sundararajan, S. P. Sakthinathan, S. Vijayakumar, K. Sathiyamoorthy, V. Mala, G. Vanangamudi, K. Thirumurthy, P. Mayavel, G. Thirunarayanan, Spectrochim. Acta. 101A (2013) 239-248.

[19] G. Thirunarayanan, International Letters of Chemistry, Physics and Astronomy 4 (2014) 109-116.

[20] W. F. Winecoff, D. W. Boykin Jr., J. Org. Chem. 37 (4) (1972) 676.

[21] A. Perjessy, M. Laucova, Coll. Czech. Chem. Commun. 36 (1971) 2944-22950.

[22] G. Thirunarayanan, M. Gopalakrishnan G. Vanangamudi, Spectrochim. Acata 67A (2007) 1106-1612.

[23] G. Thirunarayanan, M. Suresh, International Letters of Chemistry, Physics and Astronomy 4 (1 (2014) 1-11.

[24] G. Thirunarayanan, Bull. Chem. Soc. Ethiop. 28(1) (2014) 1-7.

[25] K. G Sekar, G. Thirunarayanan, International Letters of Chemistry, Physics and Astronomy 8(3) (2013) 249-258. 
[26] K. G. Sekar, G. Thirunarayanan, International Letters of Chemistry, Physics and Astronomy 8(2) (2013) 160-174.

[27] G. Thirunarayanan, G. Vanangamudi, V. Sathiyendiran, K. Ravi, Indian J. Chem. 50B(4) (2011) 593-604.

[28] S. John Joseph, R. Arulkumaran, D. Kamalakkannan, S. P. Sakthinathan, R. Sundararajan, R. Suresh, S. Vijayakumar, K. Ranganathan, N. Kalyanasundaram, G. Vanangamudi, G. Thirunarayanan, International Letters of Chemistry, Physics and Astronomy 4 (2014) 48-65.

[29] M. Subramanian, G. Vanangamudi, G. Thirunarayanan, Spectrochim Acta 110A (2013) 116-123.

[30] K. G. Sekar, G. Thirunarayanan, Int. J. Sci. Res. Know. 1(8) (2013) 299-307.

[31] G. Thirunarayanan, P. Mayavel, K. Thirumurthy, G. Vanangamudi, K. Lakshmanan, K. G. Sekar, Int. J. Chem. 1(2) (2012) 166-172.

[32] S. P. Sakthinathan, R. Suresh, V. Mala, K. Sathiyamoorthy, D. Kamalakkannan, K. Ranganathan, R. Arulkumaran, S. Vijayakumar, R. Sundararajan, G. Vanangamudi, G. Thirunarayanan, International Letters of Chemistry, Physics and Astronomy 6 (2013) 77-90.

[33] S. P. Sakthinathan, R. Suresh, V. Mala, K. Sathiyamoorthy, D. Kamalakkannan, K. Ranganathan, S. John Joseph, G. Vanangamudi, G. Thirunarayanan, Int. J. Sci. Res. Know. 1(11) (2013) 472-483.

[34] Swain C. G., Lupton E. C. Jr., J. Am. Chem. Soc. 90 (1968) 4328-4337. 